\title{
TO DEVELOP A DICOM VIEWER TOOL FOR VIEWING JPEG 2000 IMAGE AND PATIENT INFORMATION
}

\author{
Trupti Baraskar $^{1}$ and Vijay Mankar ${ }^{2}$ \\ ${ }^{1}$ Department of Electronic Engineering, SGBA, Amravati University \\ ${ }^{2}$ Vijay Mankar, MSBTE Pune, India
}

\begin{abstract}
Imaging and Communications in Medicine (DICOM) standard is an image archive system which allow itself to serve as an image manager that control the acquisition, retrieval, and distributions of medical images within entire picture archiving and communication The DICOM technology is suitable when sending images between different departments within hospitals or/and other hospitals, and consultant. However, some hospitals lack the DICOM system. In this paper proposed algorithm view and converts .dcm image files jpeg2000 standard image, whereby the image should be viewable, using with common image viewer programs. Now this files are ready to transfer via internet and easily viewable on normal computer systems using JPEG2000 viewer or on Linux platform and Windows platform.
\end{abstract}

\section{KEYWORDS}

Medical Images, DICOM, JPEG2000, JPEG2000 Compression.

\section{INTRODUCTION}

In this papers we are dealing with the DICOM (Digital Imaging and Communication in Medicine) and JPEG2000 compression technique. Here proposed work is deal with converting .dcm (DICOM) file into a JPEG2000 image format and analysing the compression size between these two image formats. Now a day's DICOM is widely used in medical field for storing images. It also a standard for handling, storing, printing, and transmitting information in medical imaging. DICOM image file format stores the details about the image and the patient's details in the same file [1]. Different medical devices produce DICOM images like MRI, CT etc. These DICOM images can be visible or readable only on DICOM viewer devices. Normal computers cannot support to DICOM image format. The National Electrical Manufacturers Association (NEMA) holds the copyright to this standard. It was developed by the DICOM Standards committee.

\subsection{DCM File}

It is a binary file, which means that an ASCII-character-based text editor like Notepad does not show it properly. A DICOM file may be encoded in Little Endian or Big Endian byte orders. Elements in a DICOM file are always in ascending order, of tags. Private tags are always odd numbered [2]. 
Signal \& Image Processing : An International Journal (SIPIJ) Vol.8, No.2, April 2017

\subsection{DICOM File Format}

A DICOM file divided into a three part i.e. Preamble, Prefix and Data Set. Preamble is an optional part of size 128 bytes. Many times it consists of a sequence of " 0 " and " 1 ". Next part is prefix which contain the four characters i.e. D, I, C and M. These characters can be used to recognise given file is DICOM file or not. After that, next part of the file is Data Set. This data Set is built from number of Data Elements. Each data element contains the information about image. Normally this image information has text (Patient) information and pixel (image) information. Patient or text information is represented in Tags format. Different Tags can be used to represent patient information. A Data Element is made up of fields. Common fields are: Data Element Tag, Value Length, and Value Field. After that, below this tag information image pixel information is present in $0 \& 1$ format [3]. The DICOM file format is shown in Fig. 1

\subsection{Challenges}

DICOM image file format stores the details about the image and the patient's details in the same file. So size of images is very large, so these images occupy large space on the storage disk. Our main target is to reduce the size of such files and to send these images over the network for consultancy purpose or for getting the expert advice from another country or state.

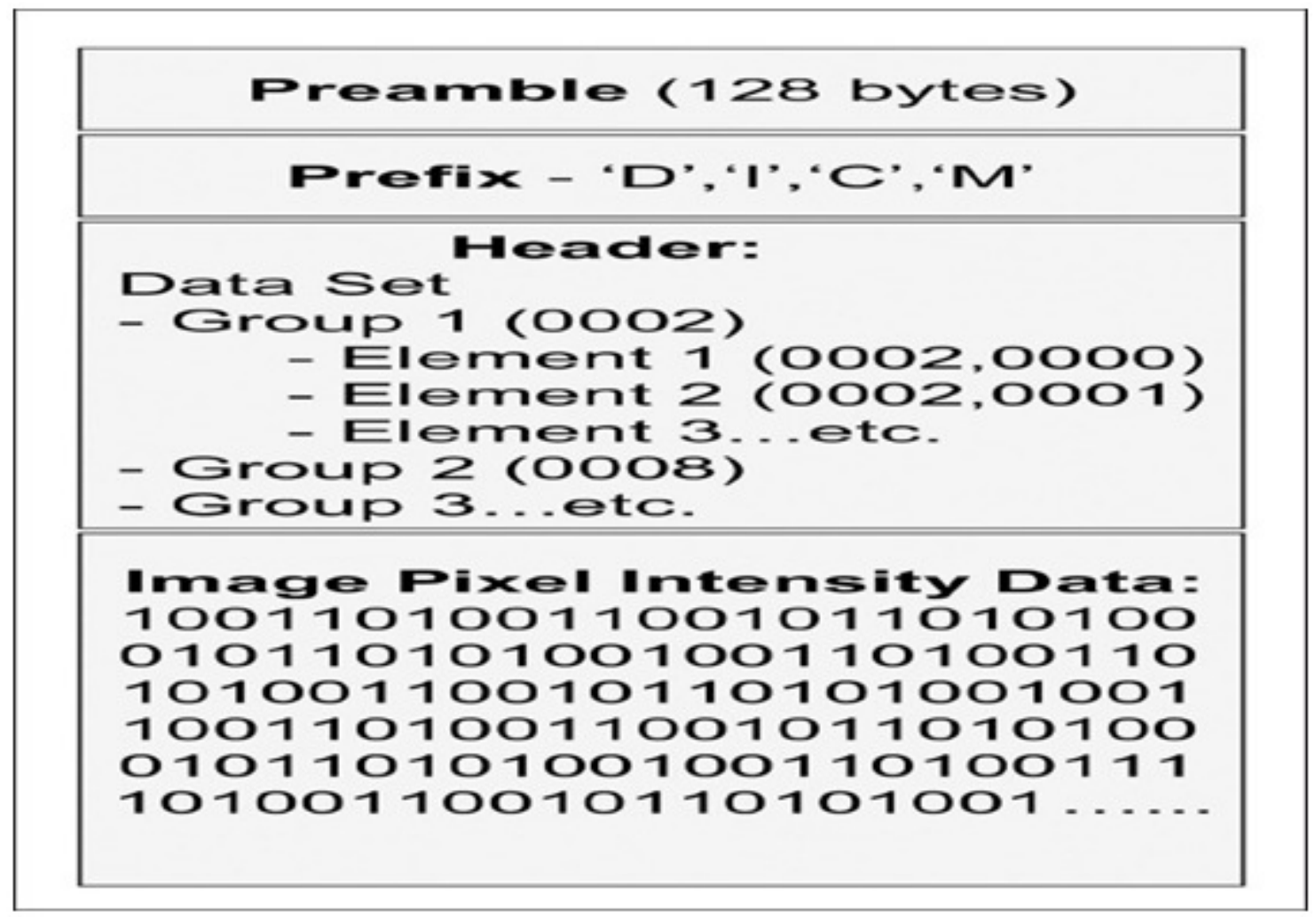

Fig. 1. DICOM File Format 
Signal \& Image Processing : An International Journal (SIPIJ) Vol.8, No.2, April 2017

\section{THEORY}

The proposed work is based on JPEG2000 as a compression technique instead of JPEG because JPEG2000 is the upcoming compression technique which has many advantages over the widely used compression technique JPEG. Here we mentioned some important limitations of JPEG compression technique:

Low bit-rate compression: JPEG offers an excellent quality at high and mid bit-rates. However, the quality is unacceptable at low bit-rates.

Lossless and lossy compression: JPEG cannot provide a superior performance at lossless and lossy compression in a single code-stream. This standard provides lossy compression with a superior performance at low bit-rates. It also provides lossless compression with progressive decoding.

Different types of still images: JPEG was optimized for natural images. Its performance on computer generated images and bi-level (text) images are poor.

Large image handling: JPEG does not allow for the compression of images larger than $64 \mathrm{~K}$ by $64 \mathrm{~K}$ without tiling. Over these limitations, JPEG2000 provides improve features which are as follows:

Protective image security: The architecture of the JPEG2000 standard makes easy the use of protection techniques of digital images such as watermarking, labeling, stamping or encryption.

Region-of-interest coding: In this, regions of interest (ROI's) can be defined. These ROI's can be encoded and transmitted with better quality than the rest of the image. Also in JPEG DCT transformation is used where in JPEG2000 DWT transformation is used [4] [5].

In addition to the DICOM format, the radiologist routinely encounters images of several file formats such as JPEG, TIFF, GIF, and PNG, BMP each format has its own unique advantages and disadvantages, which must be taken into consideration when images are archived and diagnosis. Following result identified after literature survey of view medical images stored in DICOM format and convert them to another common format [6][7].

Table1. Comparison between image formats

\begin{tabular}{|l|l|l|l|l|l|}
\hline Image Size & $\begin{array}{l}\text { Input } \\
\text { DICOM } \\
\text { Image in KB }\end{array}$ & BMP & TIFF & PNG & JPEG \\
\hline 512 by 512 & $521 \mathrm{~KB}$ & $66 \mathrm{~KB}$ & $51 \mathrm{~KB}$ & $37 \mathrm{~KB}$ & $13 \mathrm{~KB}$ \\
\hline 256 by 256 & $130 \mathrm{~KB}$ & $258 \mathrm{~KB}$ & $257 \mathrm{~KB}$ & $144 \mathrm{~KB}$ & $39 \mathrm{~KB}$ \\
\hline
\end{tabular}

\section{IMPLEMENTATION OF DICOM VIEWER}

Review Stage DICOM image file format stores the details about the image and the patient's details in the same file. So size of images is very large, and that's why we are not able to send these images over the network for consultancy purpose or for getting the expert advice from the 
Signal \& Image Processing : An International Journal (SIPIJ) Vol.8, No.2, April 2017

person who is situated in another country or state. The DICOM images require special type of viewer to view the image and it is not available everywhere.

We have developed the application that convert the .dcm file into .jp2 file format. And again this .jp2 file you can open using this viewer also. This viewer performs the following operation:

1. Extracting the patient details from the DICOM image.

2. Extracting the pixel data from the DICOM image.

3. Viewing the patient details and DICOM image separately.

4. Converting .dcm file into .jp2 file format.

4.1 Saving the patient details into text file.

4.2 Showing saved .jp2 image into separate window.

Below we are giving some important snap shots from our Image viewer, which are showing GUI, Patient data, saving image as .jp2 and comparison between .jp2 and .dcm image.

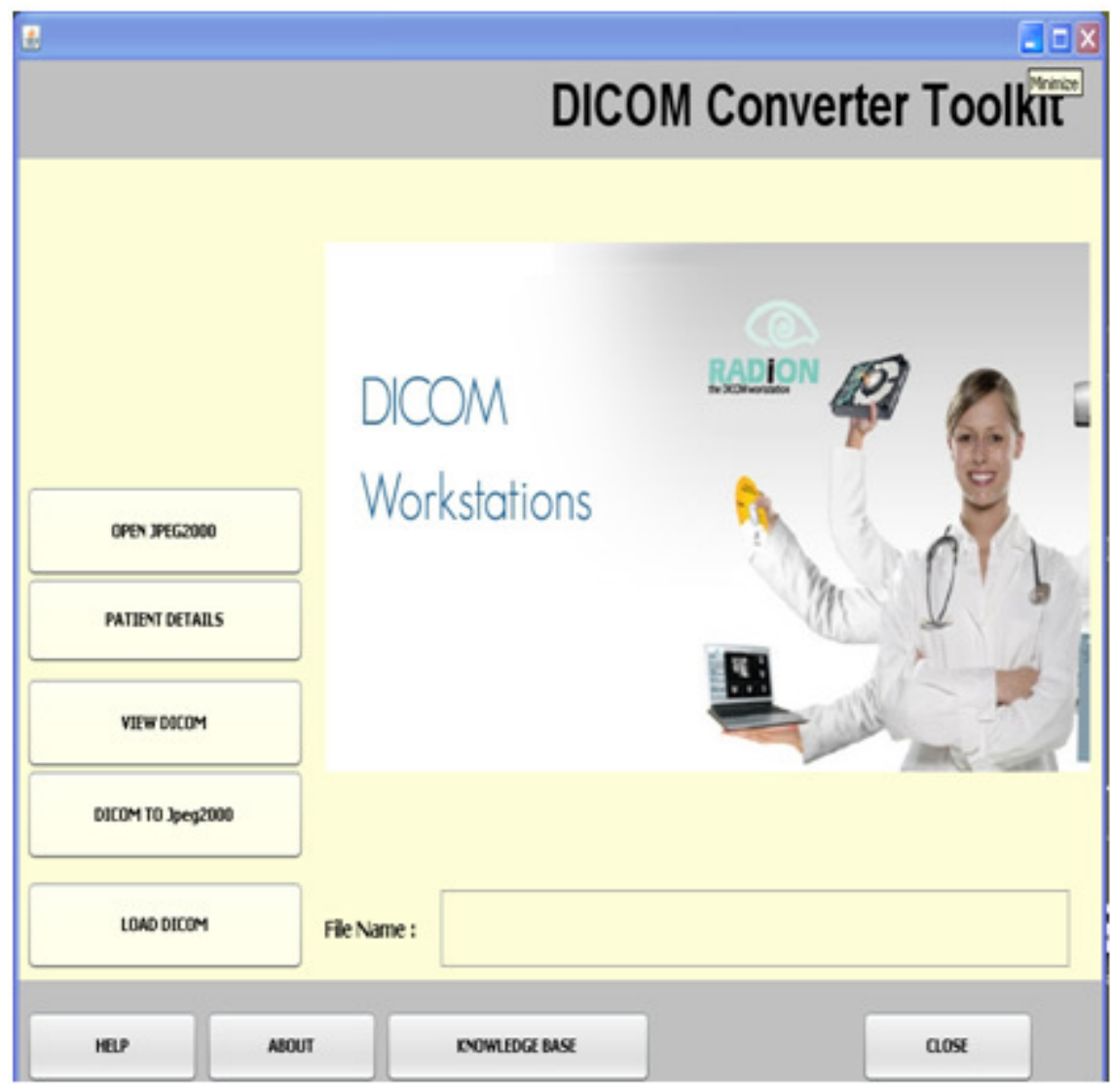

Figure2. DICOM Viewer (GUI) 
Signal \& Image Processing : An International Journal (SIPIJ) Vol.8, No.2, April 2017

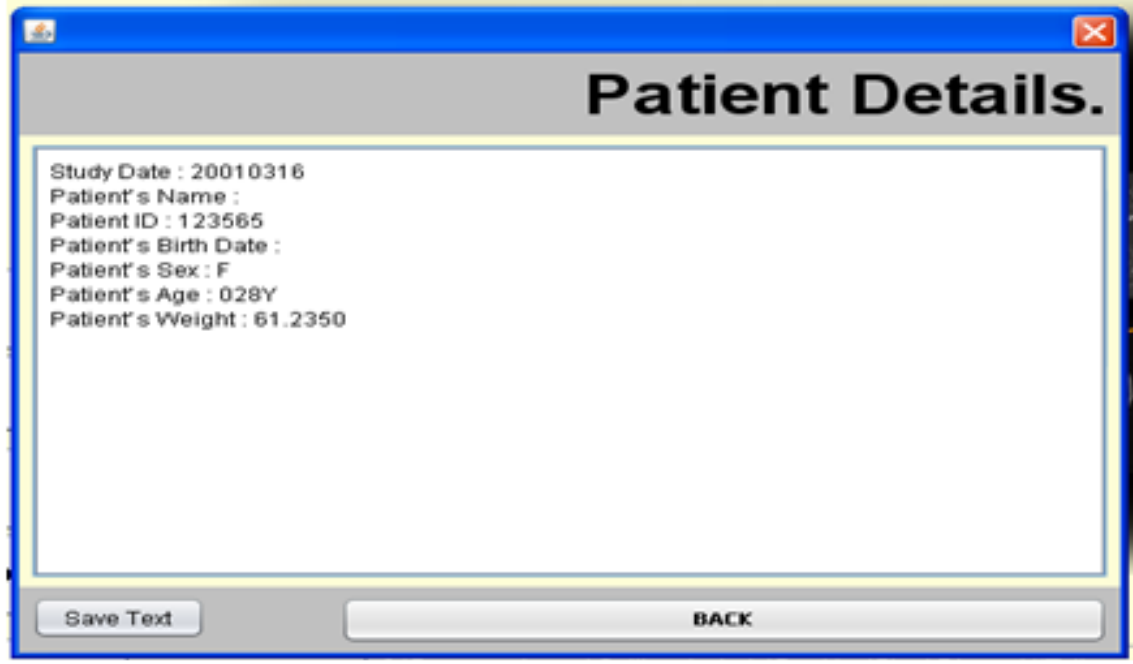

Figure3. View Patient Data

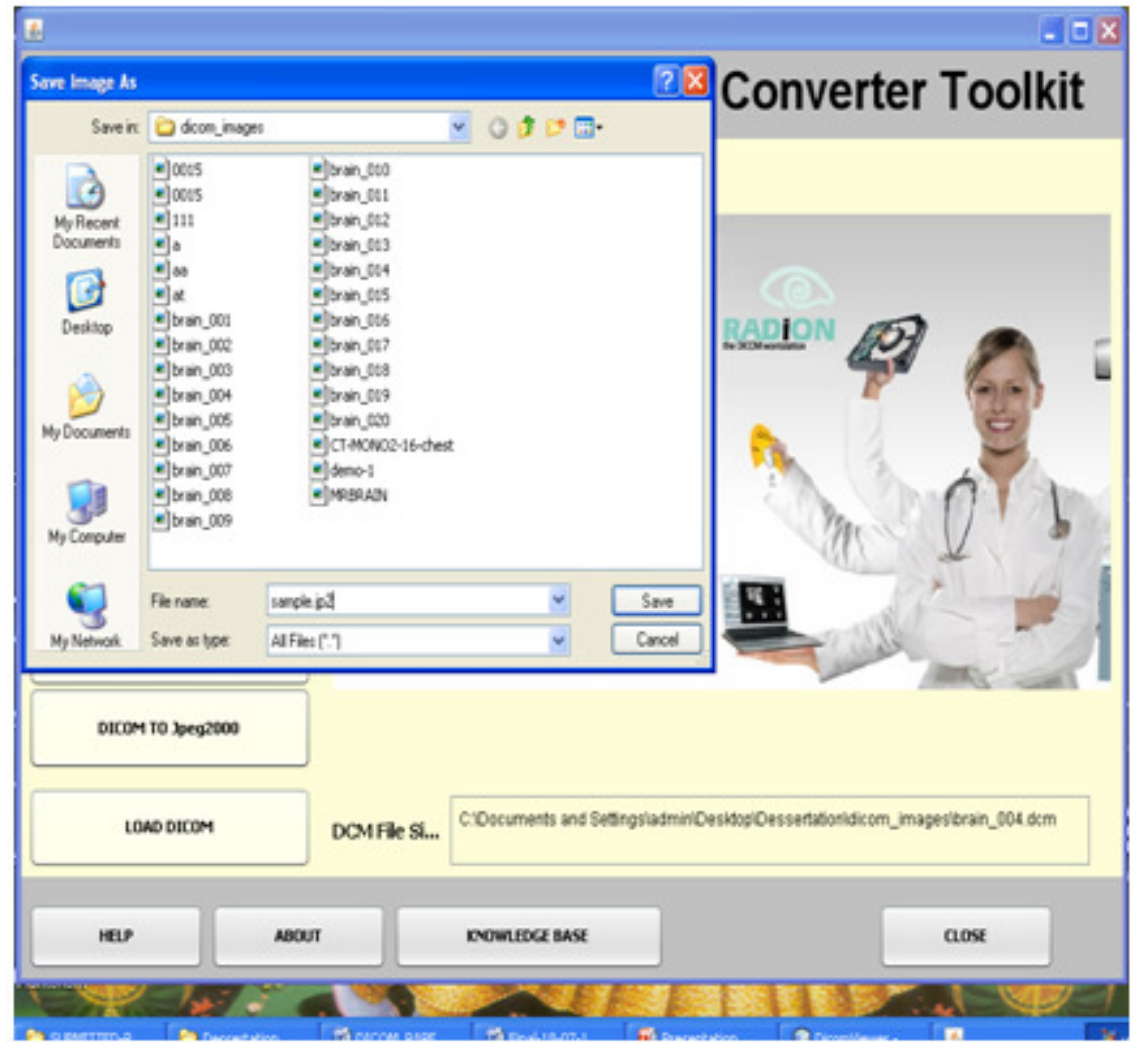

Figure 4. Save image as .jp2 
Signal \& Image Processing : An International Journal (SIPIJ) Vol.8, No.2, April 2017

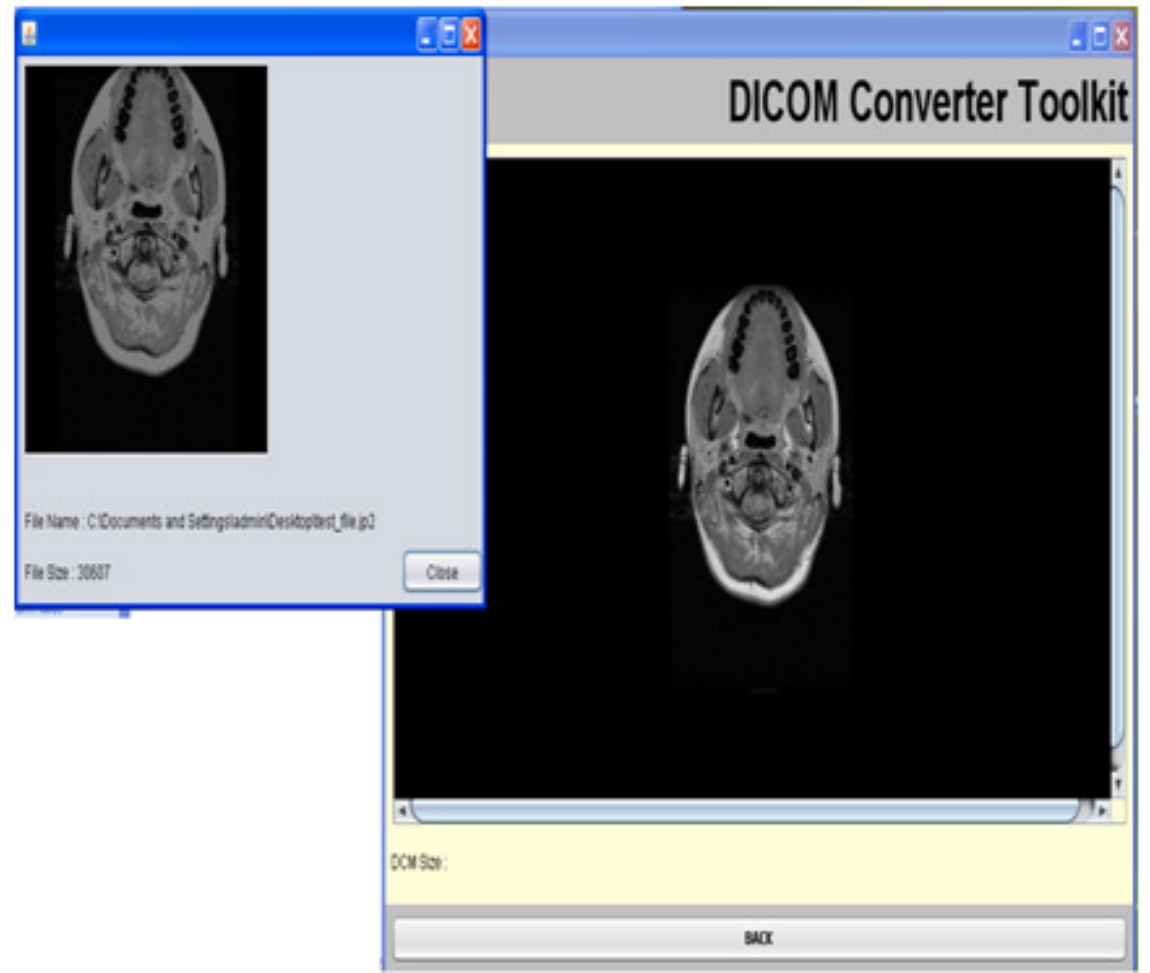

Figure 5. Comparison between .jp2 and .dcm image

\subsection{Execution Steps:}

Step 1: Select the DICOM file from the specific directory.

Step 2: Get the default parameter of file.

Step 3: Decode input stream.

Step 4: Read metadata of image.

Step 5: Read patient data and pixel data from image.

Step 6: Display the DICOM image.

Step 7: Display patient data and save into text file.

Step 8: Convert the DICOM image into JPEG2000 image formats.

Step 9: Save the converted image with .jp2 extension.

Step 10: Open .jp2 image in another window.

\section{JPEG2000 COMPRESSION METHOD}

JPEG2000 is a new compression standard for still images intended to overcome the shortcomings of the existing JPEG standard. The standardization process is coordinated by the Joint Technical Committee on Information Technology of the International Organization for Standardization (ISO)/ International Electro-technical Commission (IEC). JPEG2000 makes use of the wavelet and sub-band technologies. This process is divided into JPEG2000 Encoding and JPEG2000 Decoding. Where output of the encoding is compressed .jp2 image but this .jp2 image is not visible. To make it visible we need to perform decoding on this .jp2 image [10] [11]. 
Signal \& Image Processing : An International Journal (SIPIJ) Vol.8, No.2, April 2017

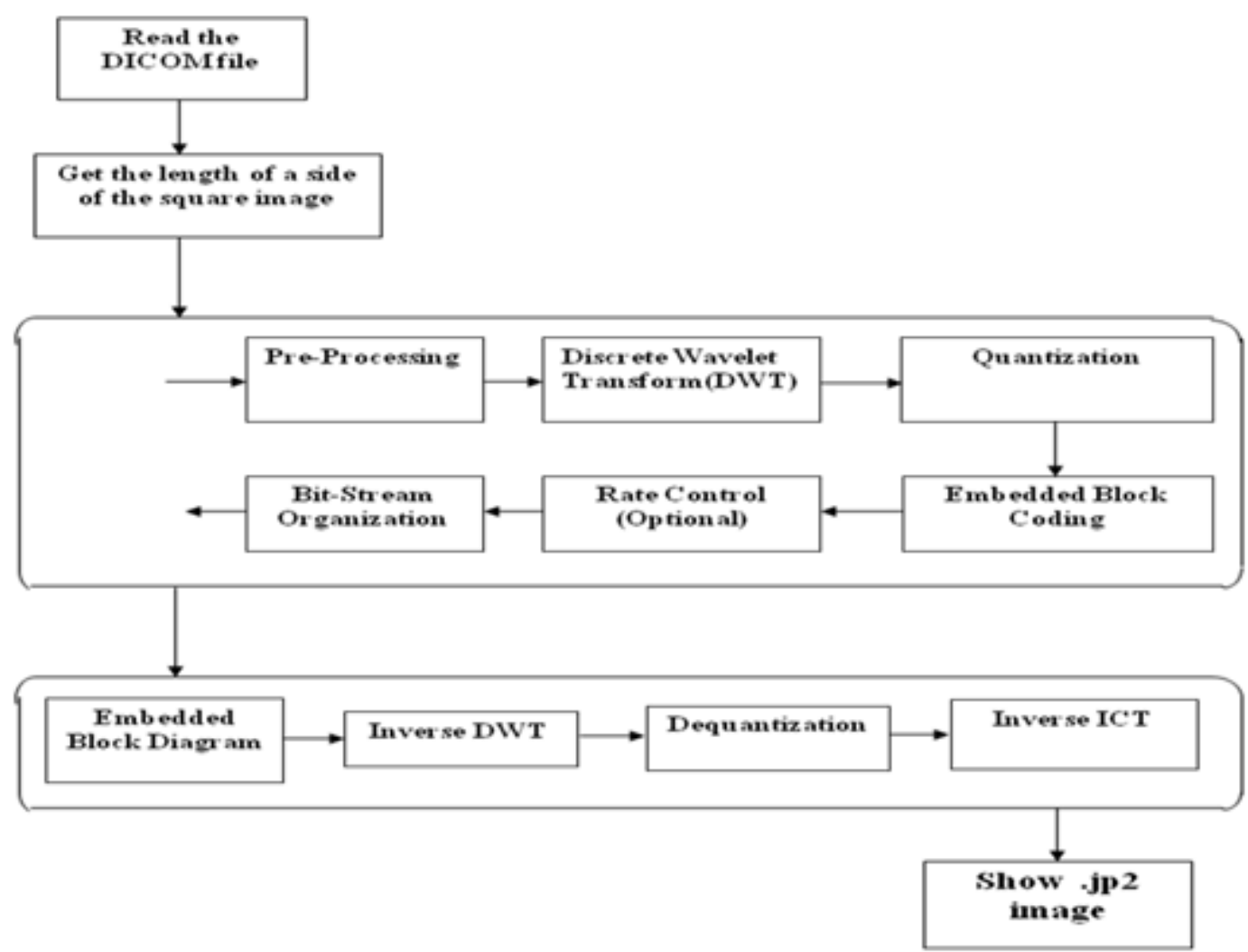

Figure 6. JPEG Compression Method

The JPEG2000 standard allows for both lossless and lossy compression. An explanation of these stages makes up the sub-sections of the encoding section.

\subsection{Pre-processing}

In the first stage, pre-processing is performed. Pre-processing actually contains three sub- stages. Shown in following diagram:

These steps must be performed so that the discrete wavelet transformation can be properly performed. The image to be encoded might be larger than the amount of memory available to the encoder. To solve this problem, JPEG2000 allows for optional tiling. In tiling, the input image is partitioned into rectangular and non-overlapping tiles of equal size (except possibly for those tiles at the image borders). Each tile is compressed independently using its own set of specified compression parameters. JPEG2000 expects its input sample data to have a nominal dynamic range centered about zero. This expectation is necessary since JPEG2000 uses high-pass filtering. The level offset pre-processing stage ensures that this expectation is met. If the original B-bit image sample values are unsigned (non-negative) quantities, an offset of $-2 \mathrm{~B}-1$ is added so that the samples have a signed representation in the range $-2 \mathrm{~B}-1<\mathrm{x}[\mathrm{n}]<2 \mathrm{~B}-1$. If the data is already signed (centered around zero), no adjustment is performed. At this point, it is important to understand the image model that JPEG2000 uses. From the standard's point of view, an image is composed of one or more components (up to 214), and each component consists of a matrix of samples representing the luminosity of the component at that point [7] 


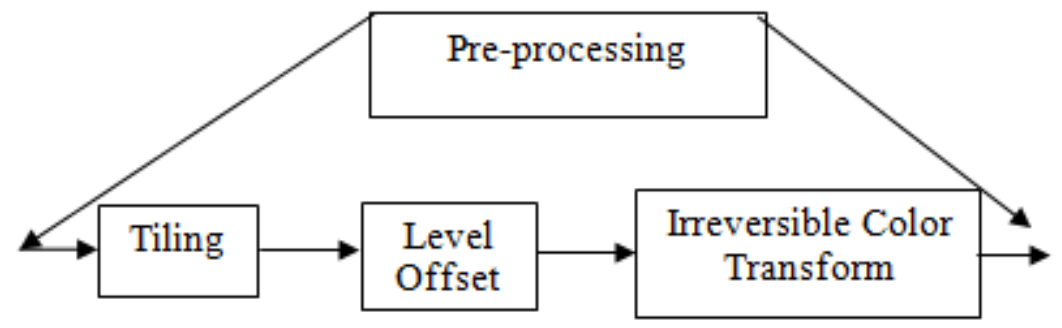

Figure7. Pre-processing sub stages

\subsection{Discrete Wavelet Transformation}

JPEG2000 uses discrete wavelet transformation to decompose each image tile into its high and low sub bands. The DWT is performed by filtering each row and column of the preprocessed image tile with a high-pass and low-pass filter. Because this process results in double the number of samples, the output from each filter is down sampled by 2 (every other value is removed) so that the sample rate remains constant. Also, it does not matter if the rows or the columns of the component matrix are filtered first. The resulting transformation is the same. In JPEG2000, multiple stages of the DWT are performed. JPEG2000 supports from 0 to 32 stages. For natural images, usually between 4 to 8 stages are used [8].

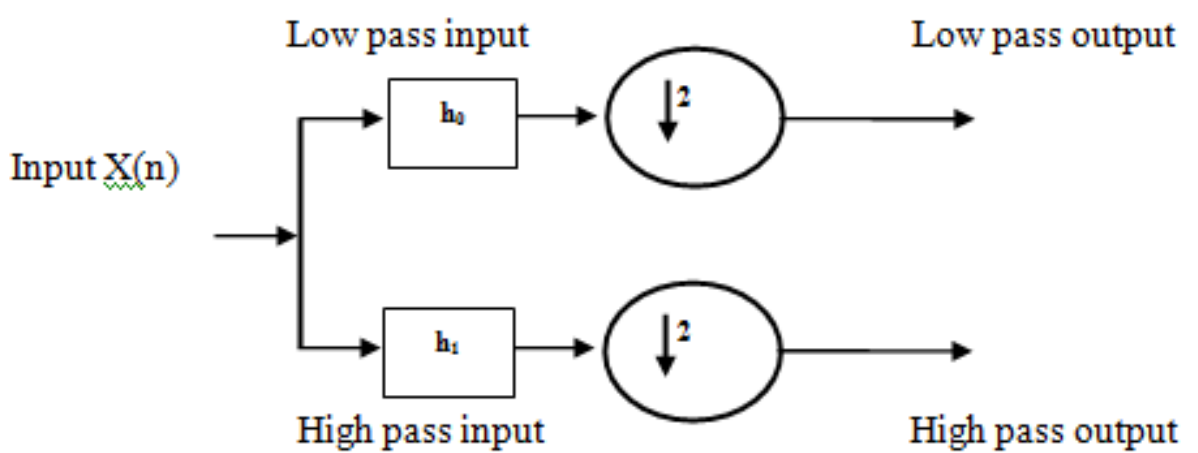

Figure 8. DWT Structure

\subsection{Quantization}

JPEG2000 performs quantization by applying the quantization function to each of the blocks of the DWT. A different quantization step size is computed for each block. Each quantization step size makes use of the base step size. This value is defined as

$$
\tau=2 R-c+i\left(1+\frac{f}{211}\right)
$$

Where $\mathrm{R}$ is the number of bits needed to represent the original intensities, $\mathrm{i}$ is the number of iterations of the DWT, and $\mathrm{c}, \mathrm{f}$ are the number of bits used to represent the exponent and mantissa, respectively of the blur portion of the DWT. We take $R=8, i=3, c=85$, and $f=8$ to obtain 
Signal \& Image Processing : An International Journal (SIPIJ) Vol.8, No.2, April 2017

$$
\tau=28-8.5+3\left(1+\frac{8}{211}\right) \approx 5.678951
$$

For the blur portion of the transform, we use the quantization step size $=\tau / 2 i=\tau / 8 \approx 0.709809$. For the detail blocks, we consider $d=\tau / 2 k-1$ for the vertical and horizontal blocks, and $d=\tau / 2(k-2)$ for the vertical blocks. Here, $\mathrm{k}=1$...i. For this example, the first iteration would use $d \approx 5.678951$ for the first iteration vertical and horizontal detail blocks and $d \approx 11.357903$ for the first iteration diagonal block. The quantization step sizes for the second iteration are 2839476, 5.678951 for the vertical/horizontal and diagonal blocks, respectively. The quantization step sizes for the third iteration are 1.419738, 0.709869 for the vertical/horizontal and diagonal blocks, respectively. Note the quantization steps sizes decrease as the iteration value increases. Note that the last vertical block and the blur block use quantization step size $0.709869<1$. Dividing by this number actually increases values in these blocks.

\subsection{Embedded Block Coding}

Embedded Block Coding tries to encode the quantized Discrete Wavelet Transform data by context-based arithmetic coding approach. After quantization, the sub-band of the 2DDWT is divided into several code-blocks that can be processed by the EBC. Each code-block is then decomposed into several bit-planes (BP). The EBC skips those insignificant bit-planes and then encodes the code block from the most significant bit-plane, bit-pane by bit-plane. EBC classifies it into three categories (passes), and the context modeling encodes the sample in three different coding passes upon its significance category. A context based adaptive binary arithmetic coder is used to compress each bit-plane in a sequence of three coding passes: significance propagation, magnitude refinement and clean-up [9].

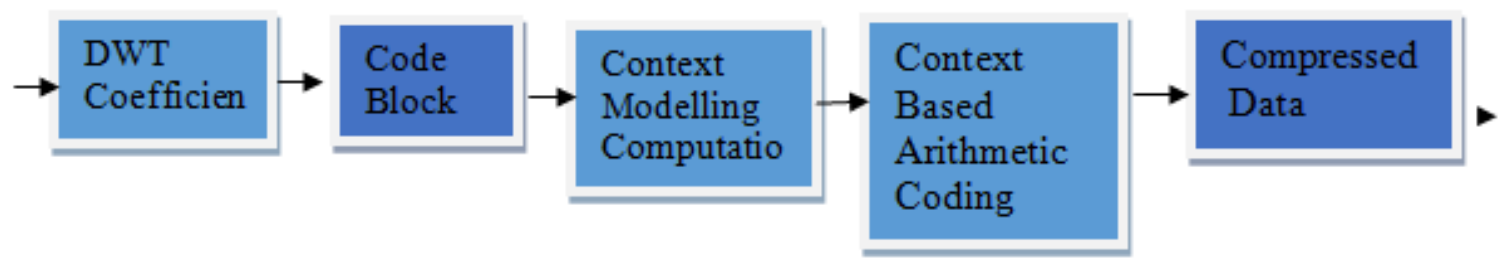

Figure.9. Functional Block of Embedded Block Coding

\subsection{Rate Control}

Rate control is the process by which the code stream is altered so that a target bit rate can be reached. Once the entire image has been compressed, a post-processing operation passes over all the compressed blocks and determines the extent to which each block's embedded bit stream should be truncated in order to achieve the target bit rate. The ideal truncation strategy is one that minimizes distortion while still reaching the target bit-rate. The overall reconstructed image distortion can be represented as a sum of the distortion contributions from each of the codeblocks. Since the code blocks are compressed independently, any bit stream truncation policy can be used. 
Signal \& Image Processing : An International Journal (SIPIJ) Vol.8, No.2, April 2017

- Which code-blocks are included in the packet?

- The number of most significant all zero bit-planes skipped by the entropy encoder for each newly included code-block

- The number of included coding passes for each code-block

- The length of included coded data for each code-block, potentially zero

\subsection{Bit-stream Organization}

In bit stream organization, the compressed data from the bit-plane coding passes are first separated into packets. One packet is generated for each precinct in a tile. A precinct is essentially a grouping of code blocks within a resolution level. Precincts divide a resolution level of a component into rectangles of size $(\mathrm{Px}, \mathrm{Py})$ containing $2 \mathrm{Px} \times 2 \mathrm{Py}$ samples. Since precincts cannot overlap code-blocks and must have dimensions that are exact powers of 2, the precinct size restricts the subordinate code-block partitions. As previously stated, each precinct generates one packet, even if the packet is empty. A packet is composed of a header and the compressed data. Then, the packets are multiplexed together in an ordered manner to form one code-stream. In Part 1 , there are five built-in ways to order the packets, called progressions, where position refers to the precinct number:

- Quality: layer, resolution, component, position

- Resolution 1: resolution, layer, component, position

- Resolution 2: resolution, position, component, layer

- Position: position, component, resolution, layer

- Component: component, position, resolution, layer

\section{RESULT ANALYSIS}

At the end of this proposed work, we find following results. Initially the size of an image is 130 $\mathrm{kb}$ of. $\mathrm{dcm}$ file which contain both header and image. This system does not take any direct input from the environment, the environment does not affect the working of the system in any way. The time taken by the proposed work is to parse the image takes a delay of one second and thus does not affect the performance of the system. GUI of this propose work is very user friendly. Initially the size of an image is $130 \mathrm{~kb}$ of .dcm file which contain both header and image. Following the compression between DICOM image size and compressed DICOM Image in to JPEG2000 and JPEG.

Table 2. Result of Compression in Percentages

\begin{tabular}{|c|c|c|c|}
\hline $\begin{array}{c}\text { Image } \\
\text { Format }\end{array}$ & $\begin{array}{c}\text { Input } \\
\text { DICOM } \\
\text { Image }\end{array}$ & $\begin{array}{c}\text { Output } \\
\text { JPEG2000 } \\
\text { Format }\end{array}$ & $\begin{array}{c}\text { Compression in } \\
\text { \% }\end{array}$ \\
\hline $\mathrm{CT}$ & $130 \mathrm{~KB}$ & $30 \mathrm{~KB}$ & 77 \\
\hline $\mathrm{MR}$ & $1026 \mathrm{~KB}$ & $88 \mathrm{~KB}$ & 91.43 \\
\hline $\mathrm{MR}$ & $514 \mathrm{~KB}$ & $44 \mathrm{~KB}$ & 91.44 \\
\hline
\end{tabular}


Signal \& Image Processing : An International Journal (SIPIJ) Vol.8, No.2, April 2017

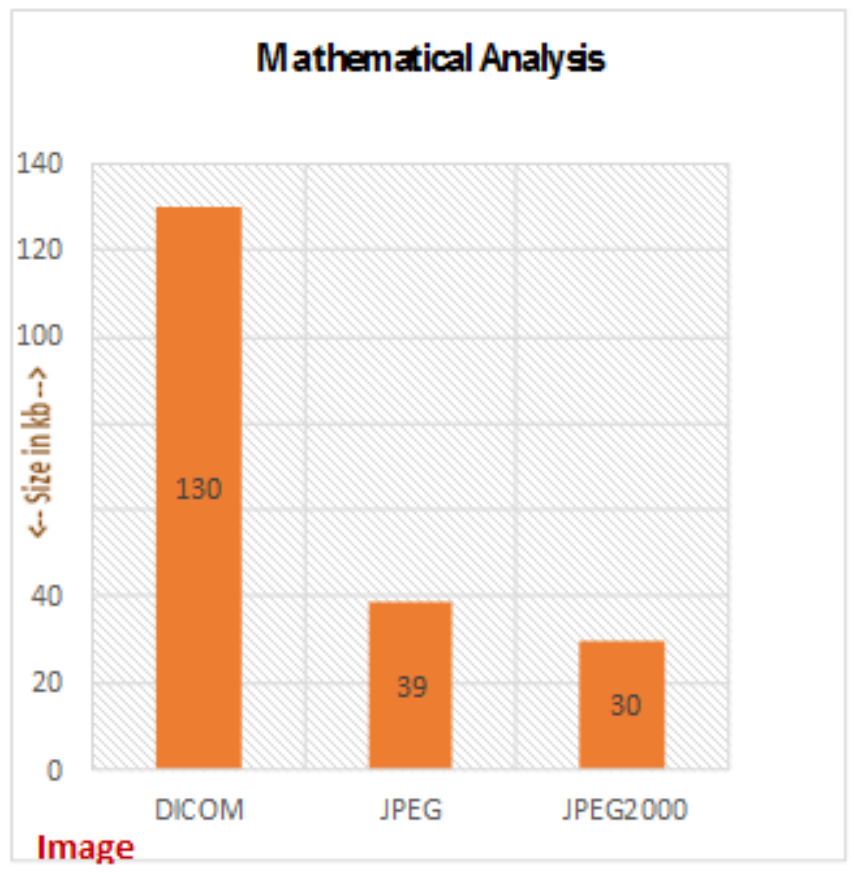

Figure10. Comparison between JPEG and JPEG2000

\section{CONCLUSION}

We come to conclusion after completing this application that the proposed system is feasible to implement conversion of DICOM images to JPEG2000 image format. DICOM Standard is developed to make possible a further expansion and easy upgrade of some parts that constantly develop. This is a very important possibility, because today imaging standards develop very fast as well as the medical imaging equipment. From the results analysis, we can conclude that jpeg2000 gives better result when compared to presently available file conversion techniques when DICOM file to any other file format. After the conversion of DICOM image to JPEG2000, it is possible to convert DICOM images into another file format using .Net tool. Handhelds have difficulty in loading and using DICOM image as they are very large and are not supported because of the limitation of their processor speed and memory. The conversion of DICOM images to jpeg2000 is carried out because it will be best support to handhelds soon.

\section{ACKNOWLEDGMENT}

Authors thank to Dr S.V. Dudal, HOD, Department of Applied Electronics, SGBA University, and Amravati, India for providing all kind of facilities and support.

\section{REFERENCES}

[1] Mario Mustra, Kresimir Delac, Mislav Grgic "Overview of DICOM Standard" 50th International Symposium ELMAR 2008, pp: 39 - 44, 10-12 September 2008, Zadar, Croatiaa 
Signal \& Image Processing : An International Journal (SIPIJ) Vol.8, No.2, April 2017

[2] Samit Desai and Usha B.S." Medical Image Transcoder for Telemedicine Based on Wireless Communication Devices", Electronics Computer Technology (ICECT), 3rd International Conference, 978-1-4244-8679-3, 2011, IEEE Explore digital library

[3] Boqiang Liu, Minghui Zhu, Zhenwang Zhang, Cong Yin, Zhongguo Liu and Jason Gu "Medical Image Conversion with DICOM", Canadian Conference on Electrical and Computer Engineering, CCECE, 2007, DOI: 10.1109/CCECE.2007.16, Print ISSN: 0840-7789, ieeexplore digital library IEEE, 2007.

[4] K. R. Rao, Y. Huh 'JPEG 2000', VIPromCom-2002,4th EURASIP - IEEE Region 8 International Symposium on Video/image Processing and Multimedia Communications, 6-19 June 2002, Zadar, Croatia.

[5] M. Rabbani and D. Santa Cruz, "The JPEG2000 Still-Image Compression Standard." Course given at the 2001 International Conference in Image Processing (ICIP), October 2001.

[6] Nitin S. Ujgare and Swati P. Baviskar "Conversion of DICOM Image in to JPEG, BMP and PNG Image Format International Journal of Computer Applications (0975 - 8887) Volume 62- No.11, January 2013

[7] Majid Rabbani*, Rajan Joshi "An overview of the JPEG2000 still image compression standard' Signal Processing: Image Communication 17 (2002) 3-48

[8] Prabhakar.Telagarapu, V.Jagan Naveen, A.Lakshmi..Prasanthi, G.Vijaya Santhi "Image Compression Using DCT and Wavelet Transformations" International Journal of Signal Processing, Image Processing and Pattern Recognition Vol. 4, No. 3, September, 2011

[9] Tsung-Da Lin, Wei-Bin Yang and Chang-Yu Hsieh "High Efficiency Concurrent Embedded Block Coding Architecture for JPEG 2000" Tamkang Journal of Science and Engineering, Vol. 13, No. 3, pp. 295304 (2010)

[10] Boqiang Liu, Minghui Zhu, Zhenwang Zhang, Cong Yin, Zhongguo Liu and Jason Gu "Medical Image Conversion with DICOM" IEEE, 2007

[11] Kyucheol Cho, Jaejoon Kim, Se-Yoon Jung, Kyuhyeon Kim, Hyun-Kook Kuhng "Development of Medical Imaging Viewer: Role in DICOM Standard”, IEEE, 2005.

\section{AUTHORS}

Trupti Baraskar obtained his Engineering Graduate Degree in Electronics and Telecommunication Engineering from Oriental Institute of Science ND Technology, Bhopal (MP) India in 2000, Post Graduate Degree in Communication Controls and Networking from Madhav Institute of Science and Technology, Gwalior (India) in 2004.

He has been in the field of teaching since last 13 years and is presently, working as an Assistant Professor in Department of Information Technology, Maharashtra Institute of Technology, Pune India. He is also working as a research scholar in S.G.B.Amravati University and is working on development of "Development of Data Integrating and Compression Techniques in DICOM Images" 
Signal \& Image Processing : An International Journal (SIPIJ) Vol.8, No.2, April 2017

Prof. Dr. Vijay R. Mankar received his B.E. degree in Electronics and Power Engineering from Government College of Engineering, Amravati (India) in 1986, M.Tech. in Electronics Engineering from erstwhile Visvesvaraya Regional College of Engineering, Nagpur (India) in 1990 and Ph.D. from S.G.B. Amravati University, Amravati (India) in 2009.

He has been in the field of teaching since last 25 years and is presently, working as a Deputy Secretary, MSBTE, Pune Region. He has served as Head of Department and the Professor, with Department of Electronics Engineering, Govt. Polytechnic, Amravati. 